\title{
Antioxidant activity of blue whiting protein hydrolysates - an in vitro study
}

\author{
S. Heffernan ${ }^{1}$, P.A. Harnedy-Rothwell ${ }^{2}$, S. Gite ${ }^{3}$, J. Whooley ${ }^{3}$, R.J. FitzGerald ${ }^{2}$ and \\ N.M. O'Brien ${ }^{1}$ \\ ${ }^{1}$ School of Food and Nutritional Sciences, University College Cork, Cork, Republic of Ireland, \\ ${ }^{2}$ Department of Biological Sciences, University of Limerick, Limerick, Republic of Ireland and \\ ${ }^{3}$ Bio-Marine Ingredients Ireland Ltd., Monaghan, Republic of Ireland
}

Prolonged oxidative stress, induced by cellular metabolic processes and/ or environmental factors, can contribute to numerous chronic non-communicable diseases including neurodegenerative diseases, cancer, diabetes, liver injury, rheumatoid arthritis, respiratory disease, and accelerated ageing ${ }^{(1)}$. This study investigated the cellular antioxidant activity of six blue whiting soluble protein hydrolysates (BWSPH, BW-SPH-A - BW-SPH-F), produced using a proprietary process, using murine RAW264.7 macrophage cells in culture.

The antioxidant activity of the BWSPHs was determined via investigating their ability to scavenge the 1-diphenyl-2-picrylhydrazyl (DPPH) radical, as well as, to inhibit the production of reactive oxygen species (ROS) and to promote the expression of endogenous antioxidant glutathione (GSH) and catalase (CAT) enzyme activity in oxidant-treated RAW264.7 cells.

Hydrolysate BW-SPH-A exhibited superior DPPH radical scavenging activity compared to the other five hydrolysates, inhibiting DPPH by $50 \%$ at a concentration of $2.10 \pm 0.12 \mathrm{mg} / \mathrm{mL}$. BW-SPH-A $(0.5 \% \mathrm{w} / \mathrm{v})$ was the only hydrolysate to significantly increase GSH and CAT levels in tert-butyl (tBOOH) and hydrogen peroxide (H2O2) challenged RAW264.7 cells, respectively, compared with the treated controls $(\mathrm{p}<0.05)$. Exposure of RAW264.7 cells to BW-SPH-A $(0.5 \% \mathrm{w} / \mathrm{v})$ for $24 \mathrm{~h}$ prior to treatment with $\mathrm{H} 2 \mathrm{O} 2$ also significantly reduced $(\mathrm{p}<0.05)$ the generation of ROS compared to cells treated with $\mathrm{H} 2 \mathrm{O} 2$ alone. Although protein hydrolysates may demonstrate promising bioactivities in vitro, efficacy may be altered in vivo due to the harsh conditions and oxidative nature of the gastrointestinal (GI) tract. The effect of in vitro GI digestion on the antioxidant activity of the BWPHs was subsequently assessed. The digestate of hydrolysate BW-SPH-A $(0.5 \% \mathrm{w} / \mathrm{v})$ also increased GSH concentration in tBOOH-treated RAW264.7 cells and reduced ROS production in RAW264.7 cells challenged with $\mathrm{H} 2 \mathrm{O} 2$ compared to treated controls $(\mathrm{p}<0.05)$.

In conclusion, it is possible that hydrolysate BW-SPH-A, which exhibited antioxidant activity in vitro, may also protect against oxidative stress in vivo and thereby, may act as a safe and natural alternative to synthetic antioxidants.

\section{Reference}

1. Droge W (2002) Physiol Rev 82, 47-95. 